\title{
A Simultaneous Iteration Algorithm for Solving Extended Split Equality Fixed Point Problem
}

\author{
Meixia Li, ${ }^{1}$ Xiping Kao, ${ }^{2}$ and Haitao $\mathrm{Che}^{1}$ \\ ${ }^{1}$ School of Mathematics and Information Science, Weifang University, Weifang, Shandong 261061, China \\ ${ }^{2}$ College of Mathematics and Systems Science, Shandong University of Science and Technology, Qingdao, Shandong 266590, China \\ Correspondence should be addressed to Meixia Li; limeixia001@163.com
}

Received 16 January 2017; Accepted 20 March 2017; Published 6 April 2017

Academic Editor: Roberto Fedele

Copyright (c) 2017 Meixia Li et al. This is an open access article distributed under the Creative Commons Attribution License, which permits unrestricted use, distribution, and reproduction in any medium, provided the original work is properly cited.

We study a kind of split equality fixed point problem which is an extension of split equality problem. We propose a kind of simultaneous iterative algorithm with a way of selecting the step length which does not need any a priori information about the operator norms and prove that the sequences generated by the iterative method converge weakly to the solution of this problem. Some numerical results are shown to confirm the feasibility and efficiency of the proposed methods.

\section{Introduction}

Let $C$ and $Q$ be nonempty closed and convex subsets of the real Hilbert spaces $H_{1}$ and $H_{2}$, respectively. The split feasibility problem is to find

$$
x \in C \quad \text { such that } A x \in Q .
$$

It can be used in various disciplines such as image restoration and radiation therapy treatment planning $[1,2]$. These applications are in finite-dimensional Hilbert spaces [3, 4]. It also can be found in an infinite-dimensional real Hilbert space $[5,6]$.

Recently, Moudafi [7] introduced a new split equality feasibility problem. Let $H_{1}, H_{2}$, and $H_{3}$ be real Hilbert spaces. Let $A: H_{1} \rightarrow H_{3}$ and $B: H_{2} \rightarrow H_{3}$ be two bounded linear operators. The split equality feasibility problem is to find

$$
\begin{aligned}
& x \in C, \\
& y \in Q \\
& \quad \text { such that } A x=B y,
\end{aligned}
$$

which allows asymmetric and partial relations between the variables $x$ and $y$. The interest is to cover many situations, for instance, applications in decomposition methods for PDEs, in game theory and in intensity-modulated radiation therapy (for short, IMRT).
Moudafi [8] introduced the simultaneous iterative method to solve the split equality feasibility problem. Furthermore, Moudafi studied the fixed point formulation to avoid using the projection. Assume $\operatorname{Fix}(T)$ and $\operatorname{Fix}(S)$ are the sets of fixed points of $T$ and $S$, respectively, where $T: H_{1} \rightarrow H_{1}$ and $S$ : $\mathrm{H}_{2} \rightarrow \mathrm{H}_{2}$ are nonlinear operators such that $\operatorname{Fix}(T) \neq \emptyset$ and $\operatorname{Fix}(S) \neq \emptyset$. So the split equality fixed point problem is to find

$$
\begin{aligned}
& x \in \operatorname{Fix}(T), \\
& y \in \operatorname{Fix}(S)
\end{aligned}
$$

such that $A x=B y$,

where $T$ and $S$ are firmly quasi-nonexpansive mappings. In order to find the solution of the split equality problem, Che and $\mathrm{Li}$ [9] proposed the following iterative algorithm:

$$
\begin{aligned}
u_{n} & =x_{n}-\gamma_{n} A^{*}\left(A x_{n}-B y_{n}\right), \\
x_{n+1} & =\alpha_{n} x_{n}+\left(1-\alpha_{n}\right) T u_{n}, \\
v_{n} & =y_{n}+\gamma_{n} B^{*}\left(A x_{n}-B y_{n}\right), \\
y_{n+1} & =\alpha_{n} y_{n}+\left(1-\alpha_{n}\right) S v_{n},
\end{aligned}
$$

and the weak convergence of the scheme (4) can also be established. Furthermore, Chang et al. [10] modified the 
iterative scheme (4) and provided a unified framework for solving this problem without using the projection. The framework is as follows.

$$
\begin{aligned}
u_{n}= & x_{n}-\gamma_{n} A^{*}\left(A x_{n}-B y_{n}\right), \\
x_{n+1}= & \alpha_{n} x_{n} \\
& +\left(1-\alpha_{n}\right)[(1-\xi) I+\xi T((1-\eta) I+\eta T)] u_{n}, \\
v_{n}= & y_{n}+\gamma_{n} B^{*}\left(A x_{n}-B y_{n}\right), \\
y_{n+1}= & \alpha_{n} y_{n} \\
& +\left(1-\alpha_{n}\right)[(1-\xi) I+\xi S((1-\eta) I+\eta S)] v_{n} .
\end{aligned}
$$

They got the following conclusion that the sequence $\left(x_{n}, y_{n}\right)$ generated by the above modification converges weakly to a solution of problem (3). Furthermore, some authors [11-14] studied the problems (1)-(3) in Banach space. They proposed effective algorithms and proved their convergence under some conditions.

Recently, He and Sun [15] studied the problem of split convex feasibility and established a strongly convergent alternating algorithm. They proposed the following iterative algorithm to find

$$
\begin{aligned}
& x \in C, \\
& y \in Q
\end{aligned}
$$

such that $A x=B y \in K$,

via the formula

$$
\begin{aligned}
\omega_{n} & =P_{K_{n}}\left(\frac{A x_{n}+B y_{n}}{2}\right), \\
K_{n} & =\left\{z \in H_{3}: h\left(\frac{A x_{n}+B y_{n}}{2}\right)\right. \\
& \left.+\left\langle\varsigma_{n}, z-\left(\frac{A x_{n}+B y_{n}}{2}\right)\right\rangle \leq 0\right\}, \\
u_{n}= & P_{C}\left(x_{n}-\xi A^{*}\left(A x_{n}-\omega_{n}\right)\right), \\
v_{n}= & P_{Q}\left(y_{n}-\xi B^{*}\left(B x_{n}-\omega_{n}\right)\right), \\
C_{n+1} & \times Q_{n+1}=\left\{(x, y) \in C_{n} \times Q_{n}:\left\|u_{n}-x\right\|^{2}\right. \\
& +\left\|v_{n}-y\right\|^{2} \leq\left\|x_{n}-x\right\|^{2}+\left\|y_{n}-y\right\|^{2}, f\left(x_{n}\right) \\
& \left.+\left\langle\mu_{n}, x-x_{n}\right\rangle \leq 0, g\left(y_{n}\right)+\left\langle\eta_{n}, y-y_{n}\right\rangle \leq 0\right\}, \\
x_{n+1} & =P_{C_{n+1}}\left(x_{1}\right), \\
y_{n+1} & =P_{Q_{n+1}}\left(y_{1}\right), \quad \forall n \in N,
\end{aligned}
$$

where $\xi \in\left(0, \min \left(1 /\|A\|^{2}, 1 /\|B\|^{2}\right)\right), \mu_{n} \in \partial f\left(x_{n}\right), \eta_{n} \in$ $\partial g\left(y_{n}\right)$, and $\varsigma_{n} \in \partial h\left(\left(A x_{n}+B y_{n}\right) / 2\right)$. In some conditions such as $\Omega=\{(x, y) \in C \times Q: A x=B y \in K\} \neq \emptyset$, they proved that the sequences $\left(x_{n}, y_{n}\right) \rightarrow(s, t),\left(u_{n}, v_{n}\right) \rightarrow(s, t)$, and $\omega_{n} \rightarrow z^{*}:=A s=B t \in K$, where $(s, t) \in \Omega$.
In this article, we study the following problem of extended split equality fixed point, which is to find

$$
\begin{aligned}
& x \in \operatorname{Fix}(G), \\
& y \in \operatorname{Fix}(M)
\end{aligned}
$$

such that $A x=B y \in K$.

When $K=H_{3}$, problem (8) is problem (3). Therefore, problem (8) is the extension of the split equality fixed point problem. We propose the simultaneous iterative algorithm for solving this problem, which avoids using the projection and the step length sequences do not depend on the operator norms $\|A\|$ and $\|B\|$. Furthermore, we prove the sequences generated by the algorithm weakly converge to a solution of the extended split equality fixed point problem. Numerical examples show the feasibility and efficiency of this algorithm.

\section{Preliminaries}

In this paper, we recall some concepts, definitions, and conclusions, which are prepared for proving our main results. We write $x_{n} \rightarrow x$ to indicate that the sequence $\left\{x_{n}\right\}$ converges weakly to $x . x_{n} \rightarrow x$ implies that $\left\{x_{n}\right\}$ converges strongly to $x$. We denote by $H_{1}$ a real Hilbert space with inner product $\langle\cdot, \cdot\rangle$ and induced norm $\|\cdot\|$.

A mapping $T: C \rightarrow C$ is called

(i) quasi-nonexpansive, if $\operatorname{Fix}(T) \neq \emptyset$,

$$
\left\|T x-x^{*}\right\| \leq\left\|x-x^{*}\right\|, \quad \forall x \in C, x^{*} \in \operatorname{Fix}(T),
$$

(ii) quasi-pseudo-contractive, if $\operatorname{Fix}(T) \neq \emptyset$,

$$
\begin{aligned}
\left\|T x-x^{*}\right\| \leq\left\|x-x^{*}\right\|+\|T x-x\| & \\
& \\
\forall x \in C, & x^{*} \in \operatorname{Fix}(T) .
\end{aligned}
$$

A mapping $P_{C}$ is said to be metric projection of $H_{1}$ onto $C$ if, for every point $x \in H_{1}$, there exists a unique nearest point in $C$ denoted by $P_{C} x$ such that

$$
\left\|x-P_{C} x\right\| \leq\|x-y\|, \quad \forall y \in C .
$$

It is well known that $P_{C}$ is a nonexpansive mapping and is characterized by the following properties:

$$
\begin{aligned}
& \left\|P_{C} x-P_{C} y\right\|^{2} \leq\left\langle x-y, P_{C} x-P_{C} y\right\rangle, \quad \forall x, y \in H_{1}, \\
& \left\langle x-P_{C} x, y-P_{C} x\right\rangle \leq 0, \quad \forall x \in H_{1}, \quad y \in C, \\
& \|x-y\|^{2} \geq\left\|x-P_{C} x\right\|^{2}+\left\|y-P_{C} x\right\|^{2}, \\
& \forall x \in H_{1}, \quad y \in C,
\end{aligned}
$$

$$
\begin{aligned}
& \left\|(x-y)-\left(P_{C} x-P_{C} y\right)\right\|^{2} \\
& \quad \geq\|x-y\|^{2}-\left\|P_{C} x-P_{C} y\right\|^{2}, \quad \forall x, y \in H_{1} .
\end{aligned}
$$


In the proof of our results, we need the following lemmas.

Lemma 1 (see [16]). Let $H$ be a real Hilbert space; then the following conclusions hold.

$$
\begin{gathered}
\|x-y\|^{2}=\|x\|^{2}+\|y\|^{2}-2\langle x, y\rangle, \\
\forall x, y \in H, \\
\|\alpha x+(1-\alpha) y\|^{2}=\alpha\|x\|^{2}+(1-\alpha)\|y\|^{2} \\
-\alpha(1-\alpha)\|x-y\|, \\
\forall x, y \in H, \alpha \in[0,1] .
\end{gathered}
$$

Lemma 2 (see [16]). Let $H$ be a real Hilbert space and $T$ : $H \rightarrow H$ be a L-Lipschitzian mapping with $L \geq 1$. Denote

$$
G:=(1-\xi) I+\xi T((1-\eta) I+\eta T) .
$$

If $0<\xi<\eta<1 /\left(1+\sqrt{1+L^{2}}\right)$, then the following conclusions hold.

(i) $\operatorname{Fix}(T)=\operatorname{Fix}(T((1-\eta) I+\eta T))=\operatorname{Fix}(G)$.

(ii) If $T$ is demiclosed at 0 , then $G$ is also demiclosed at 0 .

(iii) In addition, if $T: H \rightarrow \mathrm{H}$ is quasi-pseudo-contractive, then the mapping $G$ is quasi-nonexpansive; that is,

$$
\begin{aligned}
& \left\|G x-u^{*}\right\| \leq\left\|x-u^{*}\right\|, \\
& \qquad x \in H, u^{*} \in \operatorname{Fix}(T)=\operatorname{Fix}(G) .
\end{aligned}
$$

\section{Main Results}

In this section, we assume that

(i) $G: H_{1} \rightarrow H_{1}$ and $M: H_{2} \rightarrow H_{2}$ are two $L-$ Lipschitzian and quasi-pseudo-contractive mappings with $L \geq 1, \operatorname{Fix}(G) \neq \emptyset$, and $\operatorname{Fix}(M) \neq \emptyset$.

(ii) $H_{1}, H_{2}$, and $H_{3}$ be real Hilbert spaces. $A: H_{1} \rightarrow H_{3}$ and $B: H_{2} \rightarrow H_{3}$ are two bounded linear operators; $A^{*}$ and $B^{*}$ are their adjoint operators, respectively. Let $K \subset \mathrm{H}_{3}$ be nonempty closed convex set. We consider the extended split equality fixed point problem (8).

Theorem 3. Let $H_{1}, H_{2}$, and $H_{3}$ be real Hilbert spaces and $K$ be a closed convex level set

$$
K=\left\{x \in H_{3}: h(x) \leq 0\right\}
$$

where $h: H_{3} \rightarrow R$ is convex function which is subdifferentiable on $K$ and its subdifferentials are bounded on bounded sets. $A$ : $H_{1} \rightarrow H_{3}$ and $B: H_{2} \rightarrow H_{3}$ are two bounded linear operators with their adjoint operators $A^{*}$ and $B^{*}$, respectively, $\sigma \in(0,1)$ is a parameter controlling step length, $0<\gamma<\eta<1 /(1+$ $\left.\sqrt{1+L^{2}}\right)$, and $\left\{\alpha_{n}\right\} \subset(0,1)$. Let $\left\{x_{n}\right\},\left\{y_{n}\right\},\left\{\omega_{n}\right\},\left\{u_{n}\right\}$, and $\left\{v_{n}\right\}$ be sequences generated by

$$
\begin{aligned}
& \omega_{n}=P_{K_{n}}\left(\frac{A x_{n}+B y_{n}}{2}\right), \\
& K_{n}=\left\{z \in H_{3}: h\left(\frac{A x_{n}+B y_{n}}{2}\right)\right. \\
&\left.+\left\langle\varsigma_{n}, z-\frac{A x_{n}+B y_{n}}{2}\right\rangle \leq 0\right\}, \\
& u_{n}=x_{n}-\xi_{n} A^{*}\left(A x_{n}-\omega_{n}\right), \\
& x_{n+1}=\alpha_{n} x_{n}+\left(1-\alpha_{n}\right) T u_{n}, \\
& v_{n}=y_{n}-\xi_{n} B^{*}\left(B y_{n}-\omega_{n}\right), \\
& y_{n+1}=\alpha_{n} y_{n}+\left(1-\alpha_{n}\right) S v_{n},
\end{aligned}
$$

where

$$
\begin{aligned}
\varsigma_{n} \in \partial h\left(\frac{A x_{n}+B y_{n}}{2}\right), \\
\xi_{n} \in(0,(1-\sigma) \\
\left.\cdot \frac{\left\|A x_{n}-\omega_{n}\right\|^{2}+\left\|B y_{n}-\omega_{n}\right\|^{2}}{\left\|A^{*}\left(A x_{n}-\omega_{n}\right)\right\|^{2}+\left\|B^{*}\left(B y_{n}-\omega_{n}\right)\right\|^{2}}\right), \\
T:=(1-\gamma) I+\gamma G((1-\eta) I+\eta G), \\
S:=(1-\gamma) I+\gamma M((1-\eta) I+\eta M),
\end{aligned}
$$

where $G$ and $M$ are demiclosed at 0 . Assume $\Omega=\{(x, y) \in$ $\operatorname{Fix}(G) \times \operatorname{Fix}(M): A x=B y \in K\} \neq \emptyset$. Then $\left(x_{n}, y_{n}\right) \rightarrow$ $(t, s),\left(u_{n}, v_{n}\right) \rightarrow(t, s)$, and $\omega_{n} \rightarrow z^{*}:=A t=B s$, where $(t, s) \in \Omega$.

Proof. It is obvious that $K \subset K_{n}$ for any $n \in N$. Let $\left(x^{*}, y^{*}\right) \in$ $\Omega$; namely, $x^{*} \in \operatorname{Fix}(G), y^{*} \in \operatorname{Fix}(M)$, and $\omega^{*}=A x^{*}=$ $B y^{*} \in K$. By Lemma 1, we have

$$
\begin{aligned}
\left\|\omega_{n}-\omega^{*}\right\|= & \left\|P_{K_{n}}\left(\frac{A x_{n}+B y_{n}}{2}\right)-P_{K_{n}}\left(\omega^{*}\right)\right\|^{2} \\
\leq & \left\|\frac{1}{2}\left(A x_{n}-\omega^{*}\right)+\frac{1}{2}\left(B y_{n}-\omega^{*}\right)\right\|^{2} \\
= & \frac{1}{2}\left\|A x_{n}-\omega^{*}\right\|^{2}+\frac{1}{2}\left\|B y_{n}-\omega^{*}\right\|^{2} \\
& -\frac{1}{4}\left\|A x_{n}-B y_{n}\right\|^{2} . \\
\left\|u_{n}-x^{*}\right\|^{2}= & \left\|x_{n}-x^{*}-\xi_{n} A^{*}\left(A x_{n}-\omega_{n}\right)\right\|^{2} \\
= & \left\|x_{n}-x^{*}\right\|^{2}+\xi_{n}^{2}\left\|A^{*}\left(A x_{n}-\omega_{n}\right)\right\|^{2} \\
& -2\left\langle x_{n}-x^{*}, \xi_{n} A^{*}\left(A x_{n}-\omega_{n}\right)\right\rangle .
\end{aligned}
$$


4

Mathematical Problems in Engineering

By (20), we have

$$
\begin{aligned}
\left\|u_{n}-x^{*}\right\|^{2}= & \left\|x_{n}-x^{*}\right\|^{2}+\xi_{n}^{2}\left\|A^{*}\left(A x_{n}-\omega_{n}\right)\right\|^{2} \\
& -\xi_{n}\left\|A x_{n}-\omega^{*}\right\|^{2}-\xi_{n}\left\|A x_{n}-\omega_{n}\right\|^{2} \\
& +\xi_{n}\left\|\omega_{n}-\omega^{*}\right\|^{2} .
\end{aligned}
$$

Similarly,

$$
\begin{aligned}
\left\|v_{n}-y^{*}\right\|^{2}= & \left\|y_{n}-y^{*}\right\|^{2}+\xi_{n}^{2}\left\|B^{*}\left(B y_{n}-\omega_{n}\right)\right\|^{2} \\
& -\xi_{n}\left\|B y_{n}-\omega^{*}\right\|^{2}-\xi_{n}\left\|B y_{n}-\omega_{n}\right\|^{2} \\
& +\xi_{n}\left\|\omega_{n}-\omega^{*}\right\|^{2} .
\end{aligned}
$$

By (21), (22), (17), and (19), we have

$$
\begin{aligned}
& \left\|x_{n+1}-x^{*}\right\|^{2}+\left\|y_{n+1}-y^{*}\right\|^{2} \\
& =\left\|\alpha_{n} x_{n}+\left(1-\alpha_{n}\right) T u_{n}-x^{*}\right\|^{2} \\
& +\left\|\alpha_{n} y_{n}+\left(1-\alpha_{n}\right) S v_{n}-y^{*}\right\|^{2} \\
& =\left\|\alpha_{n} x_{n}+\left(1-\alpha_{n}\right) T u_{n}-\left(1-\alpha_{n}\right) x^{*}-\alpha_{n} x^{*}\right\|^{2} \\
& +\left\|\alpha_{n} y_{n}+\left(1-\alpha_{n}\right) S v_{n}-\left(1-\alpha_{n}\right) y^{*}-\alpha_{n} y^{*}\right\|^{2} \\
& =\alpha_{n}\left\|x_{n}-x^{*}\right\|^{2}+\left(1-\alpha_{n}\right)\left\|T u_{n}-x^{*}\right\|^{2} \\
& -\alpha_{n}\left(1-\alpha_{n}\right)\left\|x_{n}-T u_{n}\right\|^{2}+\alpha_{n}\left\|y_{n}-y^{*}\right\|^{2} \\
& +\left(1-\alpha_{n}\right)\left\|S v_{n}-y^{*}\right\|^{2}-\alpha_{n}\left(1-\alpha_{n}\right)\left\|y_{n}-S v_{n}\right\|^{2} \\
& \leq \alpha_{n}\left\|x_{n}-x^{*}\right\|^{2}+\left(1-\alpha_{n}\right)\left\|u_{n}-x^{*}\right\|^{2} \\
& -\alpha_{n}\left(1-\alpha_{n}\right)\left(1-\alpha_{n}\right)\left\|x_{n}-T u_{n}\right\|^{2}+\alpha_{n}\left\|y_{n}-y^{*}\right\|^{2} \\
& +\left(1-\alpha_{n}\right)\left\|v_{n}-y^{*}\right\|^{2}-\alpha_{n}\left(1-\alpha_{n}\right)\left\|y_{n}-S v_{n}\right\|^{2} \\
& =\alpha_{n}\left\|x_{n}-x^{*}\right\|^{2}+\left(1-\alpha_{n}\right)\left\|x_{n}-x^{*}\right\|^{2}+\left(1-\alpha_{n}\right) \\
& \text {. } \xi_{n}^{2}\left\|A^{*}\left(A x_{n}-\omega_{n}\right)\right\|^{2}-\xi_{n}\left(1-\alpha_{n}\right)\left\|A x_{n}-\omega^{*}\right\|^{2} \\
& -\xi_{n}\left(1-\alpha_{n}\right)\left\|A x_{n}-\omega_{n}\right\|^{2}+2 \xi_{n}\left(1-\alpha_{n}\right) \\
& \text { - }\left\|\omega_{n}-\omega^{*}\right\|^{2}-\alpha_{n}\left(1-\alpha_{n}\right)\left\|x_{n}-T u_{n}\right\|^{2} \\
& +\alpha_{n}\left\|y_{n}-y^{*}\right\|^{2}+\left(1-\alpha_{n}\right)\left\|y_{n}-y^{*}\right\|^{2}+\left(1-\alpha_{n}\right) \\
& \text {. } \xi_{n}^{2}\left\|B^{*}\left(B y_{n}-\omega_{n}\right)\right\|^{2}-\xi_{n}\left(1-\alpha_{n}\right)\left\|B y_{n}-\omega^{*}\right\|^{2} \\
& -\xi_{n}\left(1-\alpha_{n}\right)\left\|B y_{n}-\omega_{n}\right\|^{2}-\alpha_{n}\left(1-\alpha_{n}\right)\left\|y_{n}-S v_{n}\right\|^{2} \\
& \leq \alpha_{n}\left\|x_{n}-x^{*}\right\|^{2}+\left(1-\alpha_{n}\right)\left\|x_{n}-x^{*}\right\|^{2}+\left(1-\alpha_{n}\right) \\
& \text {. } \xi_{n}^{2}\left\|A^{*}\left(A x_{n}-\omega_{n}\right)\right\|^{2}-\xi_{n}\left(1-\alpha_{n}\right)\left\|A x_{n}-\omega^{*}\right\|^{2} \\
& -\xi_{n}\left(1-\alpha_{n}\right)\left\|A x_{n}-\omega_{n}\right\|^{2}+\xi_{n}\left(1-\alpha_{n}\right)
\end{aligned}
$$

$$
\begin{aligned}
& \cdot\left\|A x_{n}-\omega^{*}\right\|^{2}+\xi_{n}\left(1-\alpha_{n}\right)\left\|B y_{n}-\omega^{*}\right\|^{2}-\frac{1}{2} \\
& \cdot \xi_{n}\left(1-\alpha_{n}\right)\left\|A x_{n}-B y_{n}\right\|^{2}-\alpha_{n}\left(1-\alpha_{n}\right) \\
& \cdot\left\|x_{n}-T u_{n}\right\|^{2}+\alpha_{n}\left\|y_{n}-y^{*}\right\|^{2}+\left(1-\alpha_{n}\right) \\
& \cdot\left\|y_{n}-y^{*}\right\|^{2}+\left(1-\alpha_{n}\right) \xi_{n}^{2}\left\|B^{*}\left(B y_{n}-\omega_{n}\right)\right\|^{2} \\
& -\xi_{n}\left(1-\alpha_{n}\right)\left\|B y_{n}-\omega^{*}\right\|^{2}-\xi_{n}\left(1-\alpha_{n}\right) \\
& \cdot\left\|B y_{n}-\omega_{n}\right\|^{2}-\alpha_{n}\left(1-\alpha_{n}\right)\left\|y_{n}-S v_{n}\right\|^{2} \\
& =\left\|x_{n}-x^{*}\right\|^{2}+\left\|y_{n}-y^{*}\right\|^{2}+\left(1-\alpha_{n}\right) \\
& \cdot \xi_{n}^{2}\left(\left\|A^{*}\left(A x_{n}-\omega_{n}\right)\right\|^{2}+\left\|B^{*}\left(B y_{n}-\omega_{n}\right)\right\|^{2}\right) \\
& -\left(1-\alpha_{n}\right) \xi_{n}\left(\left\|A x_{n}-\omega_{n}\right\|^{2}+\left\|B y_{n}-\omega_{n}\right\|^{2}\right) \\
& -\alpha_{n}\left(1-\alpha_{n}\right)\left(\left\|x_{n}-T u_{n}\right\|^{2}+\left\|y_{n}-S v_{n}\right\|^{2}\right) \\
& -\frac{1}{2}\left(1-\alpha_{n}\right) \xi_{n}\left\|A x_{n}-B y_{n}\right\|^{2} .
\end{aligned}
$$

Notice that

$$
\begin{aligned}
& \left\|x_{n+1}-x^{*}\right\|^{2}+\left\|y_{n+1}-y^{*}\right\|^{2} \leq\left\|x_{n}-x^{*}\right\|^{2}+\| y_{n} \\
& -y^{*} \|^{2}+\xi_{n}\left(1-\alpha_{n}\right) \\
& \cdot\left[\xi_{n}\left(\left\|A^{*}\left(A x_{n}-\omega_{n}\right)\right\|^{2}+\left\|B^{*}\left(B y_{n}-\omega_{n}\right)\right\|^{2}\right)\right. \\
& \left.-\left(\left\|A x_{n}-\omega_{n}\right\|^{2}+\left\|B y_{n}-\omega_{n}\right\|^{2}\right)\right] .
\end{aligned}
$$

Thanks to

$$
\begin{aligned}
\xi_{n} & \in(0,(1-\sigma) \\
\cdot & \left.\frac{\left\|A x_{n}-\omega_{n}\right\|^{2}+\left\|B y_{n}-\omega_{n}\right\|^{2}}{\left\|A^{*}\left(A x_{n}-\omega_{n}\right)\right\|^{2}+\left\|B^{*}\left(B y_{n}-\omega_{n}\right)\right\|^{2}}\right),
\end{aligned}
$$

we obtain

$$
\begin{gathered}
\left\|x_{n+1}-x^{*}\right\|^{2}+\left\|y_{n+1}-y^{*}\right\|^{2} \\
\leq\left\|x_{n}-x^{*}\right\|^{2}+\left\|y_{n}-y^{*}\right\|^{2} .
\end{gathered}
$$

Let

$$
X_{n}\left(x^{*}, y^{*}\right)=\left\|x_{n}-x^{*}\right\|^{2}+\left\|y_{n}-y^{*}\right\|^{2} .
$$

We obtain

$$
X_{n+1}\left(x^{*}, y^{*}\right) \leq X_{n}\left(x^{*}, y^{*}\right) .
$$

This implies that $X_{n}\left(x^{*}, y^{*}\right)$ is a nonincreasing sequence; hence $\lim _{n \rightarrow \infty} X_{n}\left(x^{*}, y^{*}\right)$ exists. As a result, $\left\{x_{n}\right\}$ and $\left\{y_{n}\right\}$ are 
bounded sequences. Rewrite (23) as

$$
\begin{aligned}
& \left(1-\alpha_{n}\right) \xi_{n}\left[\left(\left\|A x_{n}-\omega_{n}\right\|^{2}+\left\|B y_{n}-\omega_{n}\right\|^{2}\right)\right. \\
& \left.\quad-\xi_{n}\left(\left\|A^{*}\left(A x_{n}-\omega_{n}\right)\right\|^{2}+\left\|B^{*}\left(B y_{n}-\omega_{n}\right)\right\|^{2}\right)\right] \\
& +\alpha_{n}\left(1-\alpha_{n}\right)\left(\left\|x_{n}-T u_{n}\right\|^{2}+\left\|y_{n}-S v_{n}\right\|^{2}\right)+\frac{1}{2}(1 \\
& \left.-\alpha_{n}\right) \xi_{n}\left\|A x_{n}-B y_{n}\right\|^{2} \leq\left\|x_{n}-x^{*}\right\|^{2}+\left\|y_{n}-y^{*}\right\|^{2} \\
& -\left(\left\|x_{n+1}-x^{*}\right\|^{2}+\left\|y_{n+1}-y^{*}\right\|^{2}\right)=X_{n}\left(x^{*}, y^{*}\right) \\
& -X_{n+1}\left(x^{*}, y^{*}\right) .
\end{aligned}
$$

Letting $n \rightarrow \infty$ and taking the limit in (29), we have

$$
\begin{array}{r}
\left\|A x_{n}-B y_{n}\right\| \longrightarrow 0, \\
\left\|T u_{n}-x_{n}\right\| \longrightarrow 0, \\
\left\|S v_{n}-y_{n}\right\| \longrightarrow 0, \\
\left\|A x_{n}-\omega_{n}\right\| \longrightarrow 0, \\
\left\|B y_{n}-\omega_{n}\right\| \longrightarrow 0 .
\end{array}
$$

Then,

$$
\begin{aligned}
\lim _{n \rightarrow \infty}\left\|u_{n}-x_{n}\right\| & =0, \\
\lim _{n \rightarrow \infty}\left\|v_{n}-y_{n}\right\| & =0, \\
\lim _{n \rightarrow \infty}\left\|x_{n+1}-x_{n}\right\| & =\lim _{n \rightarrow \infty}\left\|T u_{n}-x_{n}\right\|=0, \\
\lim _{n \rightarrow \infty}\left\|y_{n+1}-y_{n}\right\| & =\lim _{n \rightarrow \infty}\left\|S v_{n}-y_{n}\right\|=0,
\end{aligned}
$$

which imply that $\left\{x_{n}\right\}$ and $\left\{y_{n}\right\}$ are asymptotically regular. Furthermore, we get

$$
\begin{aligned}
& \lim _{n \rightarrow \infty}\left\|T u_{n}-u_{n}\right\|=0, \\
& \lim _{n \rightarrow \infty}\left\|S v_{n}-v_{n}\right\|=0 .
\end{aligned}
$$

Since $\left\{x_{n}\right\}$ and $\left\{y_{n}\right\}$ are bounded sequences, there exist weakly convergent subsequences, say $\left\{x_{n_{i}}\right\} \subset\left\{x_{n}\right\}$ such that $x_{n_{i}} \rightarrow$ $t$; also $\left\{y_{n_{i}}\right\} \subset\left\{y_{n}\right\}$ such that $y_{n_{i}} \rightarrow s$. The Opial property guarantees that the weakly subsequential limit of $\left\{\left(x_{n}, y_{n}\right)\right\}$ is unique. So we have $x_{n} \rightarrow t, y_{n} \rightarrow s$. Therefore $u_{n} \rightarrow t, v_{n} \rightarrow$ $s$. Since $G$ and $M$ are demiclosed at 0 , and from Lemma 2, by (33), we have $T t=t, S s=s$, which imply that $t \in \operatorname{Fix}(G), s \in$ $\operatorname{Fix}(M)$. Hence,

$$
(t, s) \in \operatorname{Fix}(G) \times \operatorname{Fix}(M) \text {. }
$$

Furthermore, since $A x_{n}-B y_{n} \rightarrow A t-B s$, by using the weakly lower semicontinuity of squared norm, we have

$$
\begin{aligned}
\|A t-B s\| & \leq \liminf _{n \rightarrow \infty}\left\|A x_{n}-B y_{n}\right\|=\lim _{n \rightarrow \infty}\left\|A x_{n}-B y_{n}\right\| \\
& =0
\end{aligned}
$$

that is,

$$
A t=B s .
$$

By (31), we have $\omega_{n} \rightarrow z^{*}:=A t=B s$. Now, we prove $z^{*} \in K$. We know that $\left\{\varsigma_{n}\right\}$ is bounded. There exists $\bar{M}>0$, such that $\left\|\varsigma_{n}\right\| \leq \bar{M}$, where $\bar{M}$ is a constant. Note that $\omega_{n}=P_{K_{n}}\left(\left(A x_{n}+\right.\right.$ $\left.\left.B y_{n}\right) / 2\right) \in K_{n}$, and we have

$$
h\left(\frac{A x_{n}+B y_{n}}{2}\right)+\left\langle\varsigma_{n}, \omega_{n}-\frac{A x_{n}+B y_{n}}{2}\right\rangle \leq 0 .
$$

Hence,

$$
\begin{aligned}
h\left(\frac{A x_{n}+B y_{n}}{2}\right) & \leq-\left\langle\varsigma_{n}, \omega_{n}-\frac{A x_{n}+B y_{n}}{2}\right\rangle \\
& \leq \bar{M}\left\|\omega_{n}-\frac{A x_{n}+B y_{n}}{2}\right\| .
\end{aligned}
$$

By the lower semicontinuity of $h,(38)$, and (31), we obtain

$$
\begin{aligned}
h\left(z^{*}\right) & \leq \liminf _{n \rightarrow \infty} h\left(\frac{A x_{n}+B y_{n}}{2}\right) \\
& \leq \bar{M} \liminf _{n \rightarrow \infty}\left\|\omega_{n}-\frac{A x_{n}+B y_{n}}{2}\right\|=0 .
\end{aligned}
$$

Thus $z^{*} \in K$. The proof is completed.

\section{Consequent Results}

In this section, we give some corollaries, which are easily obtained from Theorem 3.

If $G=M$, we have the following corollary.

Corollary 4. Let $H_{1}, H_{2}$, and $H_{3}$ be real Hilbert spaces and $K$ be a closed convex level set

$$
K=\left\{x \in H_{3}: h(x) \leq 0\right\},
$$

where $h: \mathrm{H}_{3} \rightarrow \mathrm{R}$ is convex function which is subdifferentiable on $K$ and its subdifferentials are bounded on bounded sets. $A: H_{1} \rightarrow H_{3}$ and $B: H_{2} \rightarrow H_{3}$ are two bounded linear operators with their adjoint operators $A^{*}$ and $B^{*}$, respectively, $\sigma \in(0,1)$ is a parameter controlling step length, $0<\gamma<\eta<$ $1 /\left(1+\sqrt{1+L^{2}}\right)$, and $\left\{\alpha_{n}\right\} \subset(0,1)$. Let $\left\{x_{n}\right\},\left\{y_{n}\right\},\left\{\omega_{n}\right\},\left\{u_{n}\right\}$, and $\left\{v_{n}\right\}$ be sequences generated by

$$
\begin{aligned}
& \omega_{n}=P_{K_{n}}\left(\frac{A x_{n}+B y_{n}}{2}\right), \\
& K_{n}=\left\{z \in H_{3}: h\left(\frac{A x_{n}+B y_{n}}{2}\right)\right. \\
&\left.+\left\langle\varsigma_{n}, z-\frac{A x_{n}+B y_{n}}{2}\right\rangle \leq 0\right\}, \\
& u_{n}=x_{n}-\xi_{n} A^{*}\left(A x_{n}-\omega_{n}\right), \\
& x_{n+1}=\alpha_{n} x_{n}+\left(1-\alpha_{n}\right) T u_{n}, \\
& v_{n}=y_{n}-\xi_{n} B^{*}\left(B y_{n}-\omega_{n}\right), \\
& y_{n+1}=\alpha_{n} y_{n}+\left(1-\alpha_{n}\right) T v_{n},
\end{aligned}
$$


where

$$
\begin{aligned}
\varsigma_{n} & \in \partial h\left(\frac{A x_{n}+B y_{n}}{2}\right), \\
\xi_{n} & \in(0,(1-\sigma) \\
& \left.\cdot \frac{\left\|A x_{n}-\omega_{n}\right\|^{2}+\left\|B y_{n}-\omega_{n}\right\|^{2}}{\left\|A^{*}\left(A x_{n}-\omega_{n}\right)\right\|^{2}+\left\|B^{*}\left(B y_{n}-\omega_{n}\right)\right\|^{2}}\right), \\
T: & =(1-\gamma) I+\gamma G((1-\eta) I+\eta G),
\end{aligned}
$$

where $G$ is demiclosed at 0 . Assume $\Omega=\{(x, y) \in \operatorname{Fix}(G) \times$ $\operatorname{Fix}(G): A x=B y \in K\} \neq \emptyset$. Then $\left(x_{n}, y_{n}\right) \rightarrow(t, s),\left(u_{n}, v_{n}\right) \rightarrow$ $(t, s)$ and $\omega_{n} \rightarrow z^{*}:=A t=B s$, where $(t, s) \in \Omega$.

If $A=B$, we have the following corollary.

Corollary 5. Let $H_{1}$ and $H_{3}$ be real Hilbert spaces and $K$ be a closed convex level set

$$
K=\left\{x \in H_{3}: h(x) \leq 0\right\},
$$

where $h: \mathrm{H}_{3} \rightarrow \mathrm{R}$ is convex function which is subdifferentiable on $K$ and its subdifferentials are bounded on bounded sets. A : $H_{1} \rightarrow H_{3}$ is a bounded linear operator with its adjoint operator $A^{*}, \sigma \in(0,1)$ is a parameter controlling step length, $0<\gamma<\eta<1 /\left(1+\sqrt{1+L^{2}}\right)$, and $\left\{\alpha_{n}\right\} \subset(0,1)$. Let $\left\{x_{n}\right\},\left\{y_{n}\right\},\left\{\omega_{n}\right\},\left\{u_{n}\right\}$, and $\left\{v_{n}\right\}$ be sequences generated by

$$
\begin{aligned}
\omega_{n} & =P_{K_{n}}\left(\frac{A x_{n}+A y_{n}}{2}\right), \\
K_{n} & =\left\{z \in H_{3}: h\left(\frac{A x_{n}+A y_{n}}{2}\right)\right. \\
& \left.+\left\langle\varsigma_{n}, z-\frac{A x_{n}+A y_{n}}{2}\right\rangle \leq 0\right\}, \\
u_{n}= & x_{n}-\xi_{n} A^{*}\left(A x_{n}-\omega_{n}\right), \\
x_{n+1} & =\alpha_{n} x_{n}+\left(1-\alpha_{n}\right) T u_{n}, \\
v_{n}= & y_{n}-\xi_{n} A^{*}\left(A y_{n}-\omega_{n}\right), \\
y_{n+1} & =\alpha_{n} y_{n}+\left(1-\alpha_{n}\right) S v_{n},
\end{aligned}
$$

where

$$
\begin{aligned}
\varsigma_{n} \in \partial h\left(\frac{A x_{n}+B y_{n}}{2}\right), \\
\xi_{n} \in(0,(1-\sigma) \\
\left.\cdot \frac{\left\|A x_{n}-\omega_{n}\right\|^{2}+\left\|B y_{n}-\omega_{n}\right\|^{2}}{\left\|A^{*}\left(A x_{n}-\omega_{n}\right)\right\|^{2}+\left\|B^{*}\left(B y_{n}-\omega_{n}\right)\right\|^{2}}\right), \\
T:=(1-\gamma) I+\gamma G((1-\eta) I+\eta G), \\
S:=(1-\gamma) I+\gamma M((1-\eta) I+\eta M),
\end{aligned}
$$

where $G$ and $M$ are demiclosed at 0 . Assume $\Omega=\{(x, y) \epsilon$ $\operatorname{Fix}(G) \times \operatorname{Fix}(M): A x=A y \in K\} \neq \emptyset$. Then $\left(x_{n}, y_{n}\right) \rightarrow$ $(s, t),\left(u_{n}, v_{n}\right) \rightarrow(s, t)$, and $\omega_{n} \rightarrow z^{*}:=A s=A t$, where $(s, t) \in \Omega$.

If $G=M$ and $A=B$, we have the following corollary.

Corollary 6. Let $H_{1}$ and $H_{3}$ be real Hilbert spaces and $K$ be a closed convex level set

$$
K=\left\{x \in H_{3}: h(x) \leq 0\right\},
$$

where $h: \mathrm{H}_{3} \rightarrow \mathrm{R}$ is convex function which is subdifferentiable on $K$ and its subdifferentials are bounded on bounded sets. $A: H_{1} \rightarrow H_{3}$ is a bounded linear operator with its adjoint operator $A^{*}$, and $\sigma \in(0,1)$ is a parameter controlling step length, $0<\gamma<\eta<1 /\left(1+\sqrt{1+L^{2}}\right)$, and $\left\{\alpha_{n}\right\} \subset(0,1)$. Let $\left\{x_{n}\right\},\left\{y_{n}\right\},\left\{\omega_{n}\right\},\left\{u_{n}\right\}$, and $\left\{v_{n}\right\}$ be sequences generated by

$$
\begin{aligned}
& \omega_{n}=P_{K_{n}}\left(\frac{A x_{n}+A y_{n}}{2}\right), \\
& K_{n}=\left\{z \in H_{3}: h\left(\frac{A x_{n}+A y_{n}}{2}\right)\right. \\
&\left.+\left\langle\varsigma_{n}, z-\frac{A x_{n}+A y_{n}}{2}\right\rangle \leq 0\right\}, \\
& u_{n}=x_{n}-\xi_{n} A^{*}\left(A x_{n}-\omega_{n}\right), \\
& x_{n+1}=\alpha_{n} x_{n}+\left(1-\alpha_{n}\right) T u_{n}, \\
& v_{n}=y_{n}-\xi_{n} A^{*}\left(A y_{n}-\omega_{n}\right), \\
& y_{n+1}=\alpha_{n} y_{n}+\left(1-\alpha_{n}\right) T v_{n},
\end{aligned}
$$

where

$$
\begin{aligned}
\varsigma_{n} & \in \partial h\left(\frac{A x_{n}+B y_{n}}{2}\right), \\
\xi_{n} \in(0,(1-\sigma) & \\
& \left.\cdot \frac{\left\|A x_{n}-\omega_{n}\right\|^{2}+\left\|B y_{n}-\omega_{n}\right\|^{2}}{\left\|A^{*}\left(A x_{n}-\omega_{n}\right)\right\|^{2}+\left\|B^{*}\left(B y_{n}-\omega_{n}\right)\right\|^{2}}\right), \\
T: & =(1-\gamma) I+\gamma G((1-\eta) I+\eta G),
\end{aligned}
$$

where $G$ is demiclosed at 0 . Assume $\Omega=\{(x, y) \in \operatorname{Fix}(G) \times$ $\operatorname{Fix}(G): A x=A y \in K\} \neq \emptyset$. Then $\left(x_{n}, y_{n}\right) \rightarrow(t, s)$, $\left(u_{n}, v_{n}\right) \rightarrow(t, s)$, and $\omega_{n} \rightarrow z^{*}:=A t=A s$, where $(t, s) \in \Omega$.

If $G=M=A=B$ is an identity operator, we have the following corollary.

Corollary 7. Let $H_{1}$ and $H_{3}$ be real Hilbert spaces and $K$ be a closed convex level set

$$
K=\left\{x \in H_{3}: h(x) \leq 0\right\},
$$

where $h: \mathrm{H}_{3} \rightarrow \mathrm{R}$ is convex function which is subdifferentiable on $K$ and its subdifferentials are bounded on bounded sets. 

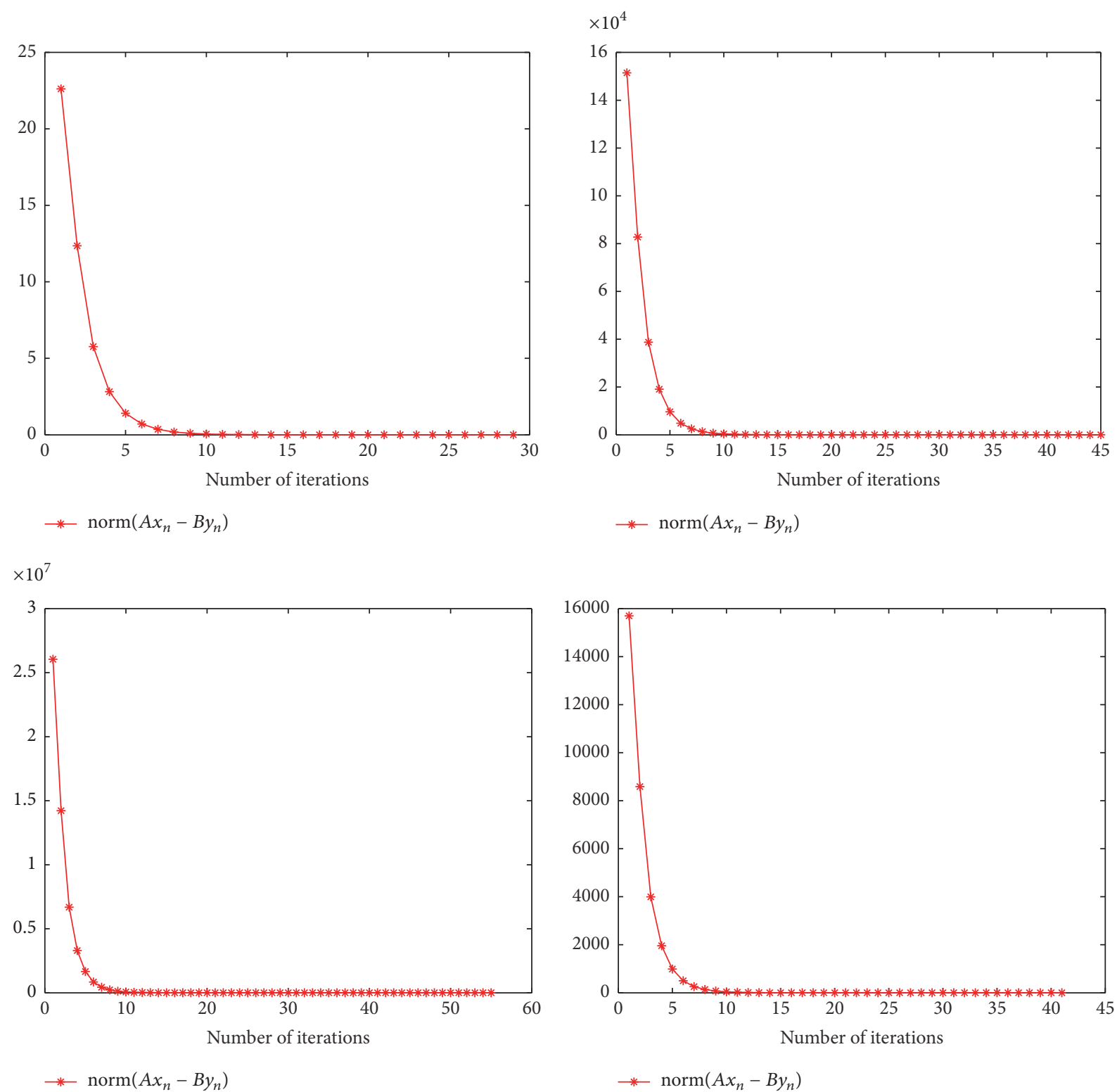

FIgURE 1: The behaviors of $\left\|A x_{n}-B y_{n}\right\|$ for different initial points.

$\sigma \in(0,1)$ is a parameter controlling step length, $0<\gamma<\eta<$ $1 /\left(1+\sqrt{1+L^{2}}\right)$, and $\left\{\alpha_{n}\right\} \subset(0,1)$. Let $\left\{x_{n}\right\},\left\{y_{n}\right\},\left\{\omega_{n}\right\},\left\{u_{n}\right\}$, and $\left\{v_{n}\right\}$ be sequences generated by

$$
\begin{aligned}
& \omega_{n}=P_{K_{n}}\left(\frac{x_{n}+y_{n}}{2}\right), \\
& K_{n}=\left\{z \in H_{3}: h\left(\frac{x_{n}+y_{n}}{2}\right)+\left\langle\varsigma_{n}, z-\frac{x_{n}+y_{n}}{2}\right\rangle\right. \\
& \quad \leq 0\} \\
& u_{n}=(1-\xi) x_{n}+\xi \omega_{n}, \\
& x_{n+1}=\alpha_{n} x_{n}+\left(1-\alpha_{n}\right) u_{n}, \\
& v_{n}=(1-\xi) y_{n}+\xi \omega_{n}, \\
& y_{n+1}=\alpha_{n} y_{n}+\left(1-\alpha_{n}\right) v_{n},
\end{aligned}
$$

where $\varsigma_{n} \in \partial h\left(\left(x_{n}+y_{n}\right) / 2\right), \xi \in(0,1-\sigma)$. Assume $\Omega=$ $\left\{(x, y) \in H_{1} \times H_{1}: x=y \in K\right\} \neq \emptyset$. Then $\left(x_{n}, y_{n}\right) \rightarrow$ $(t, s),\left(u_{n}, v_{n}\right) \rightarrow(t, s)$, and $\omega_{n} \rightarrow z^{*}:=t=s$, where $(t, s) \in \Omega$.

\section{Numerical Examples}

In this section, we give an example to show some insight into the behavior of the algorithm presented in this paper. The whole codes are written in Matlab 7.0. All the numerical results are carried out on a personal Lenovo ThinkPad computer with Intel(R) Core(TM) i7-6500U CPU $2.50 \mathrm{GHz}$ and RAM 8.00 GB.

Let $H_{1}=R^{2}, H_{2}=R^{3} . A \in R^{3 \times 2}$ and $B \in R^{3 \times 3}$ are as follows.

$$
A=\left(\begin{array}{ll}
0.8147 & 0.9134 \\
0.9058 & 0.6324 \\
0.1270 & 0.0975
\end{array}\right) \text {, }
$$


TABLE 1: Number of iterations and the CPU time for different initial points.

\begin{tabular}{lcrc}
\hline & Initial point & Iter. & Sec. \\
\hline$x=(1,1)^{T}$, & $y=(1,1,1)^{T}$ & 27 & 0.01 \\
$x=(5.2378,2.6487)^{T}$, & $y=(6.8357,43.6327,17.3853)^{T}$ & 42 & 0.016 \\
$x=(0.5221,19.0936)^{T}$, & $y=(86.1193,192.3197,152.4829)^{T}$ & 50 & 0.017 \\
$x=(0.0588,5.4403)^{T}$, & $y=(14.1190,12.9026,11.0462)^{T}$ & 38 & 0.012 \\
\hline
\end{tabular}
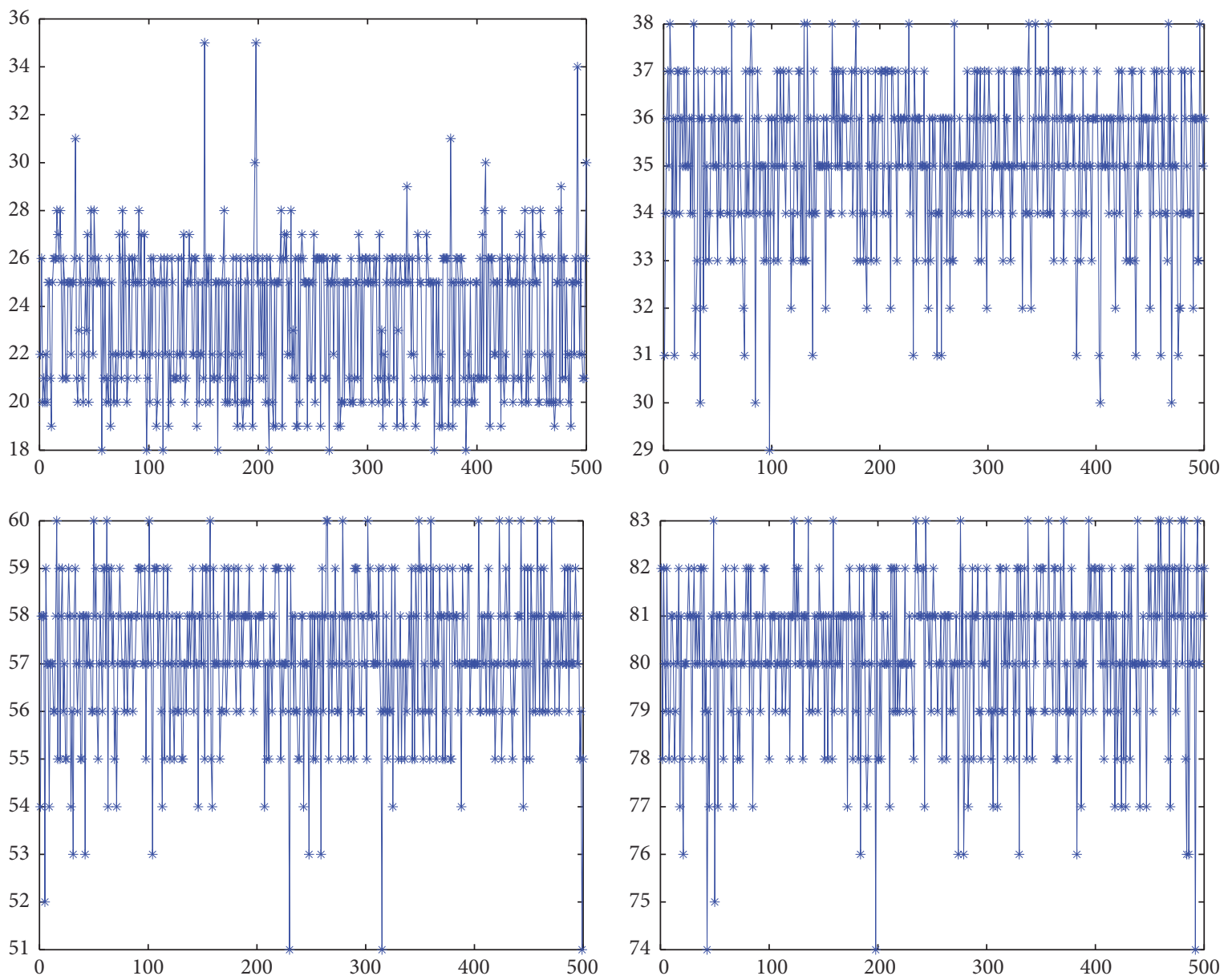

FIgURE 2: The behaviors of iterative numbers for different initial points.

$$
B=\left(\begin{array}{lll}
0.2785 & 0.9649 & 0.9572 \\
0.5469 & 0.1576 & 0.4854 \\
0.9575 & 0.9706 & 0.8003
\end{array}\right)
$$

Let $K=\left\{x \in R^{n} \mid h(x)=x_{1}^{3}+x_{2}^{2}+x_{3}-2 \leq 0\right\}$, where $x=\left(x_{1}, x_{2}, x_{3}\right)^{T}$. For convenience, we take $\sigma=0.2, \alpha_{n}=$ $1 / 3+1 / 2^{n}$, and $G=M=(1 / 2) I$. We choose $\left\|A x_{n}-B y_{n}\right\| \leq$ $10^{-6}$ as the stopping criterion.

Figure 1 presents the behaviors of $\left\|A x_{n}-B y_{n}\right\|$ in different initial points such as

$$
\begin{aligned}
& x=(1,1)^{T}, \\
& y=(1,1,1)^{T},
\end{aligned}
$$

$$
\begin{aligned}
& x=(5.2378,2.6487)^{T}, \\
& y=(6.8357,43.6327,17.3853)^{T}, \\
& x=(0.5221,19.0936)^{T}, \\
& y=(86.1193,192.3197,152.4829)^{T}, \\
& x=(0.0588,5.4403)^{T}, \\
& y=(14.1190,12.9026,11.0462)^{T} .
\end{aligned}
$$

It is easy to see that the presentation reveals that $A x=B y$. Table 1 shows the number of iterations and the CPU time 
for the above four initial points. We denote Iter. and Sec. as the number of iterations and the CPU time in seconds, respectively.

Furthermore, for testing the stationary property of iterative numbers, we carry out 500 experiments for different initial points which are presented randomly, such as

$$
\begin{aligned}
& x=\operatorname{rand}(2,1), \\
& y=\operatorname{rand}(3,1), \\
& x=10 * \operatorname{rand}(2,1), \\
& y=10 * \operatorname{rand}(3,1), \\
& x=1000 * \operatorname{rand}(2,1), \\
& y=600 * \operatorname{rand}(3,1), \\
& x=100000 * \operatorname{rand}(2,1), \\
& y=60000 * \operatorname{rand}(3,1),
\end{aligned}
$$

separately. In these cases, we take $\sigma=0.2, \alpha_{n}=0.4$ and choose $\left\|A x_{n}-w_{n}\right\|+\left\|B y_{n}-w_{n}\right\| \leq 10^{-6}$ as the stopping criterion. Figure 2 illustrates the behaviors of iterative numbers for different initial points, which reveals the stationary property of iterative numbers of the algorithm.

\section{Conflicts of Interest}

The authors declare that there are no conflicts of interest regarding the publication of this paper.

\section{Acknowledgments}

This project is supported by the Natural Science Foundation of China (Grant nos. 11401438 and 11571120) and Shandong Provincial Natural Science Foundation (Grant no. ZR2013FL032) and the Project of Shandong Province Higher Educational Science and Technology Program (Grant no. J14LI52).

\section{References}

[1] Y. Censor, T. Bortfeld, B. Martin, and A. Trofimov, "A unified approach for inversion problems in intensity-modulated radiation therapy," Physics in Medicine and Biology, vol. 51, no. 10, pp. 2353-2365, 2006.

[2] Y. Censor, A. Motova, and A. Segal, "Perturbed projections and subgradient projections for the multiple-sets split feasibility problem," Journal of Mathematical Analysis and Applications, vol. 327, no. 2, pp. 1244-1256, 2007.

[3] Q. Yang, "The relaxed CQ algorithm solving the split feasibility problem," Inverse Problems, vol. 20, no. 4, pp. 1261-1266, 2004.

[4] B. Qu and N. Xiu, "A new halfspace-relaxation projection method for the split feasibility problem," Linear Algebra and Its Applications, vol. 428, no. 5-6, pp. 1218-1229, 2008.

[5] Y. Censor, T. Elfving, N. Kopf, and T. Bortfeld, “The multiplesets split feasibility problem and its applications for inverse problems," Inverse Problems, vol. 21, no. 6, pp. 2071-2084, 2005.
[6] H.-K. Xu, "Iterative methods for the split feasibility problem in infinite-dimensional Hilbert spaces," Inverse Problems, vol. 26, no. 10, Article ID 105018, 17 pages, 2010.

[7] A. Moudafi, "Alternating CQ-algorithms for convex feasibility and split fixed-point problems," Journal of Nonlinear and Convex Analysis, vol. 15, no. 4, pp. 809-818, 2014.

[8] A. Moudafi, "A relaxed alternating CQ-algorithm for convex feasibility problems," Nonlinear Analysis, Theory, Methods and Applications, vol. 79, no. 1, pp. 117-121, 2013.

[9] H. Che and M. Li, "A simultaneous iterative method for split equality problems of two finite families of strictly pseudononspreading mappings without prior knowledge of operator norms," Fixed Point Theory and Applications, vol. 2015, article $1,2015$.

[10] S.-S. Chang, L. Wang, and L.-J. Qin, "Split equality fixed point problem for quasi-pseudo-contractive mappings with applications," Fixed Point Theory and Applications, vol. 2015, article 208, 2015.

[11] W. Takahashi, "The split feasibility problem and the shrinking projection method in banach spaces," Journal of Nonlinear and Convex Analysis, vol. 16, no. 7, pp. 1449-1459, 2015.

[12] F. Schopfer, T. Schuster, and A. K. Louis, "An iterative regularization method for the solution of the split feasibility problem in Banach spaces," Inverse Problems, vol. 24, no. 5, Article ID 055008, 2008.

[13] F. Wang, "A new algorithm for solving the multiple-sets split feasibility problem in Banach spaces," Numerical Functional Analysis and Optimization, vol. 35, no. 1, pp. 99-110, 2014.

[14] Y. Shehu, O. S. Iyiola, and C. D. Enyi, "An iterative algorithm for solving split feasibility problems and fixed point problems in Banach spaces," Numerical Algorithms, vol. 72, no. 4, pp. 835864, 2016.

[15] Z. H. He and J. T. Sun, "The problem of split convex feasibility and its alternating approximation algorithms," Acta Mathematica Sinica, English Series, vol. 31, no. 12, pp. 1857-1871, 2015.

[16] S.-S. Chang, "Some problems and results in the study of nonlinear analysis," Nonlinear Analysis, Theory, Methods and Applications, vol. 30, no. 7, pp. 4197-4208, 1997. 


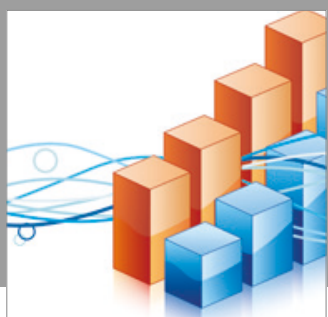

Advances in

Operations Research

vatersals

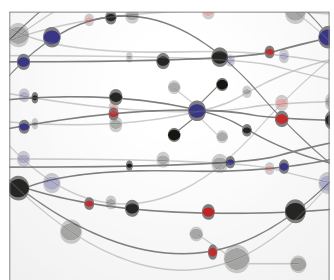

\section{The Scientific} World Journal
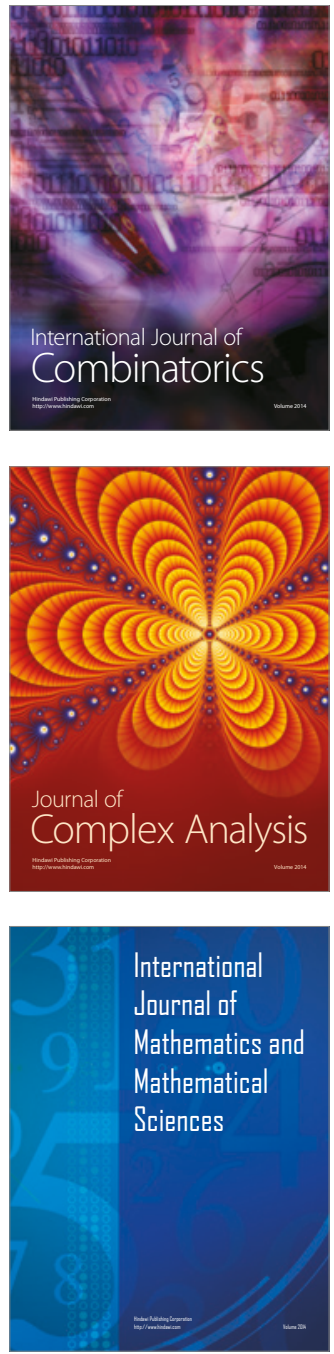
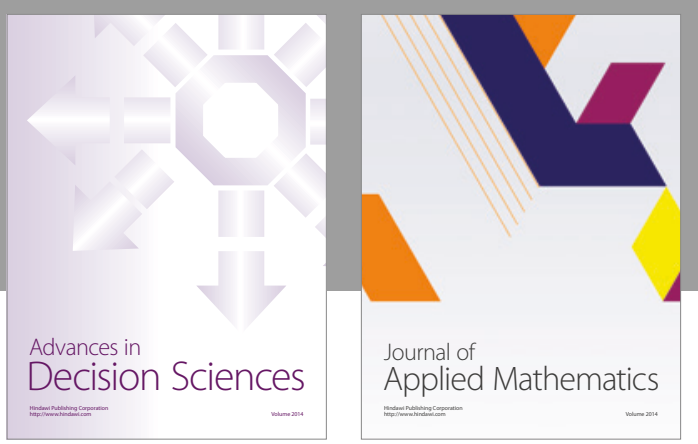

Algebra

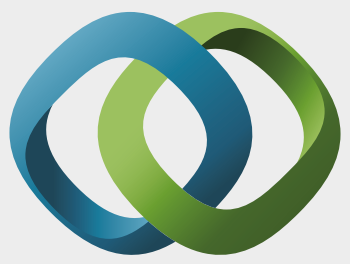

\section{Hindawi}

Submit your manuscripts at

https://www.hindawi.com
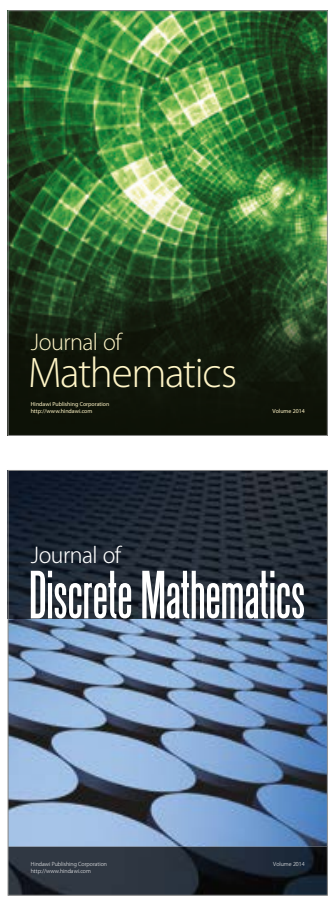

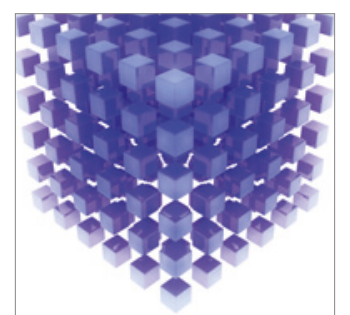

Mathematical Problems in Engineering
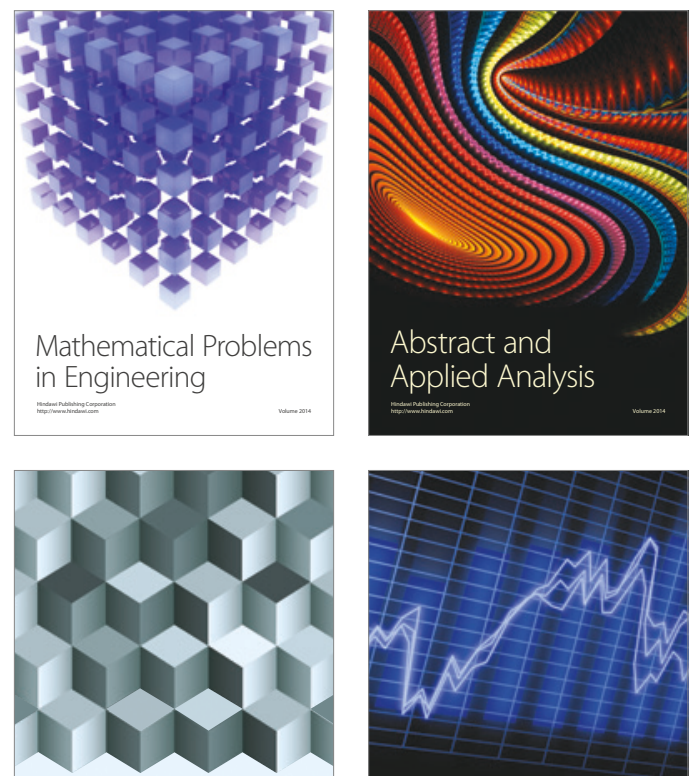

Journal of

Function Spaces

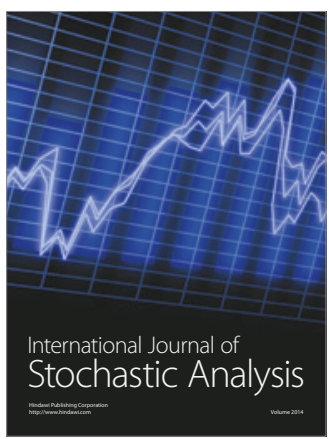

Probability and Statistics
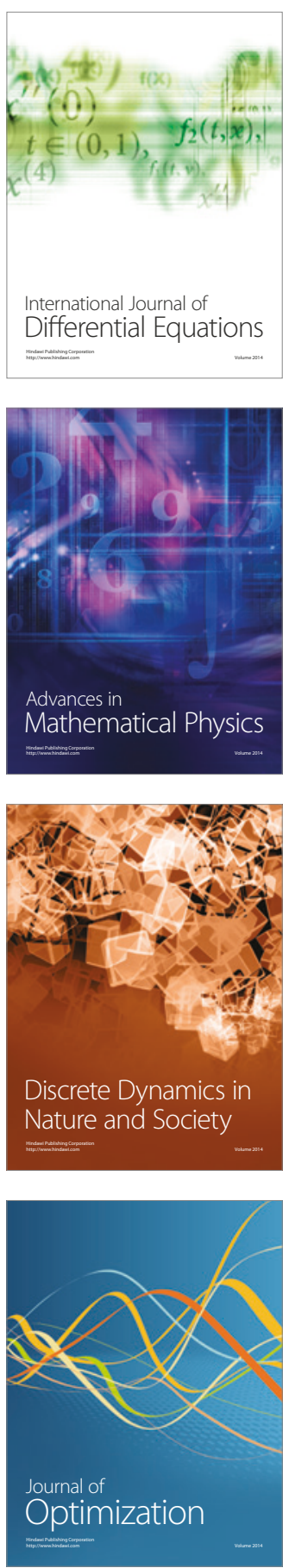